\title{
Los métodos de valuación de empresas y su relación con la capacidad de las organizaciones para generar valor
}

\section{Propuesta para reportar en la información financiera el valor de las organizaciones y su capacidad para generarlo}

\author{
Juan Alberto Adam Siade*
}

\section{Resumen}

Este artículo presenta los resultados de una investigación doctoral realizada dentro del Programa de Posgrado en Ciencias de la Administración en la Universidad Nacional Autónoma de México. La aportación principal es una propuesta para crear un nuevo estado financiero denominado Estado de Generación de Valor.

Esta propuesta puede brindar a los inversionistas y a todos los usuarios de la información elementos que los estados financieros tradicionales no contienen como la capacidad que la empresa ha tenido y tendrá para generar valor. Al incluir el valor que de la empresa se espera tener en el futuro, se cambia la tendencia tradicional en relación con los números que encontramos en los estados financieros, pues sólo representan los acontecimientos pasados.

Cabe señalar que el método de valuación de empresas que mayor consistencia tiene en su aplicación con las métricas de creación de valor económico es el método goodwill. Por otra parte, el modelo Black y Scholes no nos va a brindar el valor exacto que la empresa tendrá en el futuro, sino que - dadas las características del mismo que con bases probabilísticas sólidas indica el valor que en el futuro podría tener la empresanos señalaría si la empresa fue capaz de generar valor o no en relación con el valor que de ella misma se esperaba.

Palabras clave: valuación de empresas, modelo Black y Scholes, Valor Económico Agregado, goodwill

\footnotetext{
* Investigador de la División de Investigación de la Facultad de Contaduría y Administración, UNAM. Correo electrónico: jadam@server.contad.unam.mx
} 


\section{Introducción}

— $\mathrm{n}$ los ámbitos contable y financiero, el tema de la valuación surge a partir de la Consideración de que los informes financieros tradicionales muestran en forma limitada el valor de la empresa porque el capital contable sólo refleja información pasada. A este respecto Eccles nos dice: "Los números que encontramos en los estados financieros, como tal representan los acontecimientos pasados, pero lo que los inversionistas necesitan es otra información que les permita tomar decisiones acertadas para saber qué camino seguir." ${ }^{1}$ Es decir, los estados financieros deben complementarse con otros reportes para reflejar información que brinde más y mejores elementos que los contenidos en la información tradicional para orientar adecuadamente la toma de decisiones.

Es importante considerar que las empresas deben ser observadas como negocios en marcha, atendiendo al principio contable que lleva este nombre. Hablar de Negocio en Marcha significa señalar el antes, el ahora y el después; en este sentido, la información contable tradicional sólo se refiere al pasado y se considera que no se está cumpliendo en forma cabal con este principio, por lo que con esta investigación se desea contribuir a cumplir en forma más adecuada con la normatividad contable tanto nacional como internacional, como se señala en el apartado correspondiente.

Debido a que la información financiera contenida en los estados financieros tiene varios usuarios, el problema de no tener información más precisa de la empresa no sólo es para los inversionistas, sino que se extiende a aquellos que requieren de esta información o que toman decisiones financieras como accionistas, directores y administradores, auditores o, inclusive, clientes y la competencia. Esto significa que la información financiera tradicional no plantea el valor agregado de las empresas.

El objetivo de la presente investigación fue analizar cuáles de los métodos de valuación de empresas conocidos - método de valor en libros, método de valor de mercado, método goodwill, modelo Black y Scholes, método de flujos de efectivo descontados y método del múltiplo precio-utilidad - son más consistentes con los métodos que nos permiten medir la generación de valor como el Valor Económico

${ }^{1}$ Robert G. Eccles, et al., The value reporting revolution, p. 123 
Agregado $\left(\mathrm{EUA}^{\mathrm{TM}}\right)^{2}$; la Generación Económica Operativa $\left(\mathrm{GEO}{ }^{\circledR}\right)^{3}$; el Rendimiento Sobre la Inversión (RSI); el Rendimiento Sobre la Inversión Operativa Neta $($ RION®); la Utilidad Antes de Intereses, Impuestos, Depreciaciones y Amortizaciones (UAIIDA) y el rendimiento bursátil al ser aplicados en empresas mexicanas que cotizan en bolsa.

Una vez que se conoce esta consistencia, se podrá brindar tanto a ejecutivos como a empresarios elementos suficientes para que al aplicar cierto método de valuación de empresas cuenten con los elementos necesarios para medir la capacidad de generar valor. Para los efectos de esta investigación entenderemos por consistencia la relación lineal o correlación que existe entre las variables del estudio. También entenderemos la relación que existe cuando se observa una tendencia similar en la comparación que se hace de estas dos variables que son los métodos y las métricas bajo estudio. Esta consistencia brindó los elementos para proponer la creación de un nuevo estado financiero.

Este trabajo maneja dos variables:

- Las que consideran los métodos de valuación de empresas: método de valor en libros, método de valor de mercado, método goodwill, modelo Black y Scholes, método de flujos de efectivo descontados y método del múltiplo precio-utilidad.

- Las que consideran las métricas de creación de valor: EVA, GEO, RSI, RION, UAIIDA y el rendimiento bursátil.

Dado que se estudia el valor de las empresas y la creación de valor en el transcurso del tiempo, se analizaron los estados financieros a fechas de cierre en serie continua de los años 1992-1997, 1997-2002 y 1992-2002 de las empresas de los sectores de alimentos, bebidas y tabaco; comercio; comunicaciones; construcción; controladoras; industria de la transformación y otros servicios. Al inicio de esta investigación formaban parte de la muestra del Índice de Precios y Cotizaciones (IPC) de la Bolsa Mexicana de Valores que son comparables en todos los casos al existir y cotizar todas ellas en los años señalados. Estas empresas por emisora son: ALFA, APASCO, BIMBO, CEMEX, COMERCI, CONTAL, DESC, FEMSA,

\footnotetext{
${ }^{2}$ El EVA es una marca registrada por Stern Stewart \& Co.

${ }^{3}$ GEO y RION son marcas registradas por la Sociedad Panamericana de Estudios Empresariales, A.C.
} 
GCARSO, GISSA, ICA, KIMBER, PEÑOLES, SAVIA, SORIANA, TAMSA, TELMEX, TELEVISA y WALMEX. ${ }^{4}$

Las 19 emisoras reúnen las características de comparabilidad en el periodo estudiado de 1992 a 2002; es decir, son las que han cotizado en todo el periodo, pues para los cálculos de algunos de los métodos en 1992 se toman datos desde 1987. Además, representan el $30.64 \%$ de las empresas emisoras que actualmente operan $\left(62^{5}\right.$ de las $160^{6}$ registradas en la Bolsa Mexicana de Valores).

Con base en la información financiera de las empresas se calcularon sus valores según cada uno de los métodos de valuación y de las métricas de valor económico para descubrir estadística y analíticamente la consistencia entre ellos.

Se hizo un análisis financiero comparativo de la relación que tienen estos valores entre sí para conocer su consistencia a través de correlaciones, variaciones, tendencias y porcentajes. El análisis se realizó en dos aspectos: ${ }^{7}$

1. Análisis principales:

- Análisis de los coeficientes de correlación.

- Análisis gráfico de tendencias.

- Análisis crítico de la relación entre modelos y métricas.

- Análisis de la observación de resultados obtenidos en el cálculo de los métodos y métricas.

Se considera que el análisis de los coeficientes de correlación es el más adecuado y principal para el objetivo perseguido, dado que lo que se busca es encontrar una

\footnotetext{
${ }^{4}$ No se incluye el sector financiero por los cambios continuos que ha tenido en la última década, lo que hace imposible dar un seguimiento en las cifras de los estados financieros. Diversas instituciones que fueron adquiridas o que se fusionaron con otras cambiaron radicalmente o incluso desaparecieron. Esto provoca que existan grupos financieros con información financiera muy recientes por su actual creación y que sus cifras hayan cambiado drásticamente de un momento a otro. Todo esto provoca que sus características no sean constantes con las empresas que forman parte del estudio y, por lo tanto, su información no pueda ser comparable.

${ }^{5}$ Bolsa Mexicana de Valores (BMV), “emisoras”, México, www.bmv.com.mx, 10 de junio de 2004.

${ }^{6}$ Idem.

${ }^{7}$ Los anexos con los resultados numéricos y las gráficas no se presentan en este artículo porque son más de 120 cuartillas.
} 
relación lineal entre las variables para proponer un método que permita integrar a los estados financieros información sobre su valor económico y la capacidad para generarlo.

2. Análisis de apoyo o complementarios:

- Análisis estadístico de prueba de rechazo de la hipótesis nula de la igualdad de medias, mediante el estadístico $F$ o estadístico de prueba del análisis de varianza ANOVA

- Análisis estadístico de prueba de rechazo de la hipótesis nula mediante la distribución $t$ de student

- Análisis de significancia “ $p$ ” para determinar la validez de la prueba

Además, el tipo de estudio fue correlacional, exploratorio y longitudinal. Correlacional porque se mide la relación existente entre las variables bajo estudio; exploratorio porque se examina un tema poco estudiado en nuestro país con el enfoque de este trabajo; y longitudinal porque se estudia la evolución de las empresas con datos de 1987 a 2002.

Una vez que se determinó cuáles de los métodos de valuación son los más consistentes con las métricas de creación de valor, se realizó una propuesta para integrarlos a la información financiera de las empresas.

De acuerdo con la problemática señalada, fue importante considerar la forma cómo esta información puede integrarse a los estados financieros de las empresas para que revelen una información más confiable sobre el valor de las mismas; así, se propone la creación de un nuevo estado financiero. Para ello, se analizaron tanto las Normas Internacionales de Información Financiera como la normatividad contable mexicana para determinar cómo este estudio contribuye a cubrir la necesidad de mejorar la información que brindan los estados financieros.

Esta investigación podría contribuir a resolver la problemática, de carácter mundial, que nos dice que los inversionistas y, en general, los usuarios de la información financiera necesitan no sólo información histórica, sino también información acerca del futuro de la organización que les permita tomar decisiones acertadas. 


\section{Resultados}

\section{A. Método goodwill}

En este apartado se presentan los resultados observados en el método goodwill en relación con los tipos de análisis que se aplicaron.

El método de valuación de empresas que mayor consistencia tiene en su aplicación con las métricas de creación de valor económico es el goodwill. Es importante destacar que este método, calculado tanto con el rendimiento operativo como con el rendimiento neto, presenta la misma relación en los resultados, por lo que, después de observarlos y a diferencia de lo que se creyó en un principio, se determinó que no es necesario diferenciarlos para los efectos de esta investigación. Esto significa que cuando las empresas aplican el método goodwill para calcular su valor con cualquiera de los dos rendimientos están midiendo la capacidad que la empresa tiene para generar valor económico o para destruirlo.

El análisis que se presenta en estos resultados se refiere al goodwill operativo, pero se hacen referencias al goodwill neto o tradicional, donde se observan las similitudes en la relación de ambos con las métricas de creación de valor, con lo que se aclara por qué sólo se le denomina goodwill.

Con respecto al análisis de coeficientes de correlación se observa lo siguiente. El goodwill tiene dos etapas referentes a dos criterios de aplicación del método. El primero con el valor de la empresa arrojado por el mismo (método de valuación de empresas) y el segundo con el resultado directo del rendimiento obtenido por encima o por debajo de lo mínimo esperado (métrica de creación de valor).

En el cuadro 1 se observa la alta correlación que existe entre el valor de la empresa calculado con el primer criterio de aplicación (GODOPER) del método goodwill y el RION, el RSI y la UAIIDA presentados en pesos. 


\section{Cuadro 1}

Correlación entre el valor de la empresa calculado con el método goodwill y el rendimiento en pesos del RION, el RSI y la UAIIDA

\begin{tabular}{|l|r|r|r|}
\hline & \multicolumn{1}{|c|}{92} & \multicolumn{1}{c|}{97} & \multicolumn{1}{c|}{2002} \\
\hline & GODOPER & GODOPER & GODOPER \\
\hline RION\$ & 0.976 & 0.975 & 0.869 \\
\hline RSI\$ & 0.97 & 0.707 & 0.825 \\
\hline UAIIDA\$ & 0.979 & 0.987 & 0.864 \\
\hline
\end{tabular}

Fuente: elaboración propia

Con el segundo criterio de aplicación (GOD\$OP) del método goodwill, que se refiere al resultado directo del rendimiento por encima o por debajo de lo mínimo esperado, se observa en el cuadro 2 que existe una alta correlación con el EVA calculado con los dos criterios de referencia, el de la tasa libre de riesgo (TLR) y el del CAPM, pero no así en todos los casos con la GEO, que se calculó con los mismos criterios que el EVA.

\section{Cuadro 2}

Correlación entre el rendimiento en pesos, medido con el método goodwill, y las métricas de creación de valor EVA y GEO

\begin{tabular}{|l|r|r|r|}
\hline & \multicolumn{1}{|c|}{92} & \multicolumn{1}{|c|}{97} & \multicolumn{1}{c|}{2002} \\
\hline & GODSOP & \multicolumn{1}{c|}{ GODSOP } & \multicolumn{1}{c|}{ GOD\$OP } \\
\hline EVASTLR & 0.852 & 0.836 & 0.995 \\
\hline EVASCAPM & 0.964 & 0.94 & 0.989 \\
\hline GEOSTLR & & 0.551 & 0.885 \\
\hline GEOSCAPM & & 0.787 & 0.987 \\
\hline
\end{tabular}

Fuente: elaboración propia

De igual forma, el goodwill neto (tradicional) en el cuadro 3 presenta las mismas características de correlación que el goodwill operativo. 


\section{Cuadro 3 \\ Correlación entre el rendimiento en pesos, medido con el método goodwill neto (tradicional), y las métricas de creación de valor EVAy GEO}

Fuente: elaboración propia

Cabe aclarar que el único de los métodos aplicados que presentó una relación importante con el EVA fue el goodwill, cuadros 2 y 3.

En los cuadros 1, 2 y 3 se observa que el goodwill, con respecto al valor de la empresa, se relaciona en forma importante con el RSI, el RION y la UAIIDA, mientras que el resultado de aumento o disminución de valor del propio goodwill se relaciona con el EVA.

Esto se ejemplifica de la siguiente manera para hacer más clara su comprensión:

Supóngase que la empresa tiene un valor de los activos netos de $\$ 15,000$, que presenta un rendimiento operativo mínimo aceptable de $\$ 1,000$ y que en el cálculo del goodwill se observa un rendimiento de $\$ 1,500$. Esto significa que la empresa en los últimos cinco años ha generado, en promedio, un rendimiento por encima de lo mínimo aceptable esperado de $\$ 500$, que es la cantidad que sobrepasa dicho rendimiento. De igual forma, el valor de la empresa es de $\$ 15,500$, que es la cantidad que se suma de los propios $\$ 500$ a los activos netos. En este caso, los $\$ 15,500$ (primer criterio de aplicación) son los que se relacionan en forma importante con el ROI, el RION y la UAIIDA, mientras que los \$500 (segundo criterio de aplicación) son los que se relacionan con el EVA.

La relación que se observa en el cuadro 2 se confirma en el análisis gráfico de tendencias, en donde se aprecia que el goodwill, calculado con el segundo criterio, sigue la misma tendencia que el EVA 1 (TLR) y el EVA 2 (CAPM). Asimismo, en el cuadro 4 se confirma lo que se observa en el cuadro 2, que muestra una mayor 
relación del goodwill con el EVA que con la GEO. Este cuadro muestra el porcentaje de valores negativos en las empresas bajo estudio; en él se observa que el resultado del goodwill es más cercano, en porcentaje, a los resultados de destrucción de valor del EVA que a los de la GEO.

\section{Cuadro 4}

\section{Comparativo en porcentaje de los resultados negativos del EVA, la GEO y} el goodwill calculado con el segundo criterio de aplicación

\begin{tabular}{|c|c|}
\hline EVA 1 & $54.38 \%$ \\
\hline EVA 2 & $63.15 \%$ \\
\hline GEO 1 & $77.19 \%$ \\
\hline GEO2 & $70.17 \%$ \\
\hline GOODWILL OP. & $52.63 \%$ \\
\hline
\end{tabular}

Fuente: elaboración propia

Como se ve en el cuadro 5, el valor de mercado es el que en promedio valora más alto a las empresas, seguido del método de múltiplo precio-utilidad, ambos por arriba del valor en libros. Inferior a este valor están el método goodwill, el modelo de Black y Scholes 2 (dividendo equivalente al rendimiento real de la tasa libre de riesgo), Black y Scholes 1 (porcentaje real de los dividendos erogados) y el de flujos descontados como el método que menor valor le otorga a las empresas.

\section{Cuadro 5}

Ponderación de los métodos de valuación de empresas ordenados por el valor que arrojan

\begin{tabular}{|c|c|c|c|c|c|c|c|}
\hline Método & $\begin{array}{c}\text { VALOR } \\
\text { DE } \\
\text { MERCADO }\end{array}$ & $\begin{array}{c}\text { MÚTIPLO } \\
\text { P/U }\end{array}$ & $\begin{array}{c}\text { VALOR EN } \\
\text { LIBROS }\end{array}$ & GOODWILL & B Y S 2 & B Y S 1 & $\begin{array}{c}\text { FLUJOS } \\
\text { DESC. }\end{array}$ \\
\hline Puntuación & 325 & 279 & 269 & 261 & 229 & 222 & 68 \\
\hline Porcentaje & $19.66 \%$ & $16.88 \%$ & $16.27 \%$ & $15.79 \%$ & $13.85 \%$ & $13.43 \%$ & $4.12 \%$ \\
\hline
\end{tabular}

Fuente: elaboración propia 
En este cuadro, los valores del primer renglón son los puntos que obtuvo cada método en función del lugar que ocupó en la valuación de cada empresa; el segundo renglón son los mismos datos, pero expresados en porcentaje. Este orden se corrobora con el análisis gráfico de tendencias donde se observa que el orden de los métodos según el valor que le otorgan a las empresas coincide con este cuadro.

Es importante observar que el hecho de que el goodwill esté por debajo del valor en libros coincide con el dato en el que el EVA en la mayoría de los casos presenta datos negativos. El método goodwill aumenta o disminuye el valor en libros en función, también, de la generación de rendimiento por encima o por debajo del mínimo aceptable esperado. Con esta observación se corrobora aún más la relación entre este método de valuación y el EVA.

Este último análisis también coincide con lo que se ha dicho anteriormente en relación con el estudio de correlación en el que el goodwill tiene una alta correlación con el EVA (1) TLR, con el EVA (2) CAPM y con el estudio gráfico de tendencias.

En el análisis continuo de variaciones por los periodos 92-97, 97-02 y 92-02 se observa en el cuadro 6 que, de los métodos de valuación, el goodwill es el que más alto índice de correlación presenta con el valor en libros, lo que podría ser considerado como un factor importante dentro del análisis porque al relacionarse con dicho valor su información podría ser incluida en los estados financieros.

\section{Cuadro 6}

Correlación por series continuas de dos periodos de 5 años y uno de 10, del método goodwill con los métodos de valuación de empresas considerados en el estudio

\begin{tabular}{|l|r|r|r|}
\hline & \multicolumn{1}{|c|}{$92-97$} & \multicolumn{1}{c|}{ 97-02 } & \multicolumn{1}{c|}{ 92-02 } \\
\hline & GOODWILL & GOODWILL & GOODWILL \\
\hline VLIBROS & 0.838 & 0.799 & 0.85 \\
\hline MULT.PU & & 0.543 & 0.43 \\
\hline VMERCADO & & 0.574 & 0.292 \\
\hline B\&SDIVPA & 0.521 & 0.219 & 0.582 \\
\hline B\&SDIVTL & 0.497 & 0.265 & 0.651 \\
\hline FLUJOSDE & & 0.724 & 0.677 \\
\hline
\end{tabular}

Fuente: elaboración propia 
Por lo que respecta a las pruebas de hipótesis relacionadas con este método, no se rechaza la hipótesis nula de igualdad de varianzas (prueba $F$ ) entre el goodwill y el RSI, el RION, la UAIIDA, el EVACAPM y la GEOCAPM. Tampoco se rechazan las hipótesis nulas de igualdad de medias (prueba $t$ ) entre el goodwill y las métricas de creación de valor EVACAPM, GEOCAPM, RION, RSI y UAIIDA. Al no poderse rechazar las hipótesis nulas se puede concluir que las medias y las varianzas son iguales.

En cuanto al análisis de significancia $p$, se observó que en las pruebas de hipótesis con los estadísticos $F$ y $t$, donde las hipótesis nulas fueron rechazadas, los niveles de significancia $p$ fueron muy bajos, mientras que en las que la hipótesis nula no pudo ser rechazada la significancia $p$ fue alta. Lo anterior nos permite corroborar la igualdad de medias en los métodos que las presentaron, además de la igualdad de varianzas.

\section{B. Modelo de Black y Scholes}

Por las características observadas del comportamiento del modelo de Black y Scholes calculado con el criterio de dividendos equivalente al rendimiento real de la tasa libre de riesgo, se puede afirmar que aunque el valor que arroja el modelo no es el que la empresa tendrá en el futuro, sí puede ser considerado como elemento de referencia en cuanto a que si el valor en libros que la empresa obtenga en el futuro es inferior al valor esperado la organización no generó el valor que como mínimo se esperaba obtener o, por el contrario, si el valor en libros que la empresa obtenga en el futuro es superior al valor esperado quiere decir que generó cierto valor que rebasó las expectativas.

También se puede afirmar que el modelo de Black y Scholes, calculado con el criterio mencionado, es más acertado que los flujos de efectivo descontados aplicado con los criterios ya señalados; asimismo, ambos métodos son comparables porque tanto Black y Scholes, con bases probabilísticas sólidas, como el modelo de flujos de efectivo, con base en proyecciones, son los únicos que se adelantan a lo que la empresa valdrá en el futuro. En este sentido, el modelo de flujos de efectivo descontados comparado con el modelo de Black y Scholes no fue acertado por lo observado en los resultados obtenidos según los criterios de aplicación. 
Para los efectos de esta investigación, el problema de las proyecciones se subsanó por la misma naturaleza de la investigación que al manejar flujos descontados por cinco años para calcular el valor de las empresas, se tomaron los flujos reales generados por las mismas. Por esto se observa que hay valores negativos para la empresa al aplicar este método de valuación. Para calcular el valor de la empresa en 1992 se tomaron los flujos descontados que la empresa generó de 1993 a 1997; para el cálculo del valor de la empresa en 1997 se tomaron en cuenta los flujos de efectivo que la empresa efectivamente generó de 1998 a 2002. Al tomar los flujos que la empresa generó, se observó que muchos de ellos fueron negativos; es decir, en lugar de haber un incremento en el efectivo, se generó un decremento en el mismo, lo que provocó que, en sentido estricto de aplicación, la empresa tuviera un valor negativo calculado con este método.

Esta investigación al tomar las cifras que realmente se presentaron en las empresas elimina el elemento subjetivo, lo que permite analizar los resultados y compararlos en la forma pura y objetiva que permite sacar conclusiones más apegadas a la realidad de cada método y modelo.

Los resultados obtenidos del estudio muestran la vulnerabilidad de este método debido a que al observarlos vemos que al aplicar los flujos reales, que muchas veces fueron negativos, dieron como resultado valores negativos para las empresas, lo que en un sentido estricto es imposible, pues incluso los valores en libros también podrían ser negativos cuando los pasivos fueran superiores a los activos; pero comparándolo con los demás métodos que con solidez financiera le asignan valores positivos a las empresas - el método de flujos de efectivo descontados con las características de aplicación que se siguieron en esta investigación - es más bien un elemento de referencia que un elemento de valor "negativo" para una negociación de compra o de venta; esto es, nos puede servir para decir que la empresa tiende a valer menos porque su valor por el método de flujos es negativo que decir que ése es su valor de base para una operación de compra o de venta.

Por otro lado, en el análisis de los coeficientes de correlación también se observa que el modelo de Black y Scholes (2) (dividendo equivalente al rendimiento real de la tasa libre de riesgo) presenta correlaciones importantes en relación con las métricas presentadas en pesos del RION, RSI y UAIIDA (cuadro 7) y con los métodos de valor en libros, goodwill, múltiplo precio-utilidad y valor de mercado. Cabe destacar que con el que más se relaciona es con el goodwill (cuadro 8). 


\section{Cuadro 7}

Correlación entre el modelo Black y Scholes con el criterio de dividendo equivalente al rendimiento real de la tasa libre de riesgo, y las métricas de creación de valor RION, RSI y UAIIDA

\begin{tabular}{|l|r|r|r|}
\hline & \multicolumn{1}{|c|}{1992} & \multicolumn{1}{c|}{1997} & \multicolumn{1}{c|}{2002} \\
\hline & B\&SDIVTL & B\&SDIVTL & B\&SDIVTL \\
\hline RION\$ & 0.748 & 0.977 & 0.875 \\
\hline RSI\$ & 0.718 & 0.669 & 0.8 \\
\hline UAIIDA\$ & 0.753 & 0.981 & 0.877 \\
\hline
\end{tabular}

Fuente: elaboración propia

\section{Cuadro 8}

Correlación entre el modelo Black y Scholes con el criterio de dividendo equivalente al rendimiento real de la tasa libre de riesgo, y los métodos de valuación de empresas considerados en el estudio

\begin{tabular}{|l|r|r|r|}
\hline & \multicolumn{1}{|c|}{1992} & \multicolumn{1}{c|}{1997} & \multicolumn{1}{c|}{2002} \\
\hline & B\&SDIVTL & B\&SDIVTL & B\&SDIVTL \\
\hline VLIBROS & 0.794 & 0.969 & 0.908 \\
\hline GOODWILL & 0.839 & 0.98 & 0.928 \\
\hline MULT.PU & 0.694 & 0.912 & 0.84 \\
\hline VMERCADO & 0.654 & 0.922 & 0.85 \\
\hline FLUJOSDC & 0.388 & 0.543 & \\
\hline
\end{tabular}

Fuente: elaboración propia

Asimismo, este análisis nos permite concluir que este modelo calculado con el criterio mencionado, en relación con el dividendo equivalente al rendimiento real de la tasa libre de riesgo, puede ser considerado como un elemento de referencia importante del valor que la empresa puede tener en el futuro. Para los efectos de esta investigación se calculó el valor que el modelo Black y Scholes le asignaba a las empresas en los años 1992, 1997 y 2002 considerando cifras reales, para compararlo con los valores en libros que en realidad obtuvieron las mismas. Es decir, es un modelo que nos indica - en función del tiempo, que puede ser desde un día hasta varios años - cuál será el valor de compra, en este caso de la empresa, en 
el futuro. En este sentido el criterio de aplicación fue cuestionarnos ¿cuánto valdrá la empresa en 1992, 1997 y 2002 si calculamos su valor 5 años antes?, lo que permitió compararlo con el valor que la empresa tuvo en la realidad en esos mismos años observando una correlación importante no sólo con el valor en libros, sino también con las métricas y métodos que ya se han mencionado.

En el análisis comparativo en el mismo modelo Black y Scholes 1 (porcentaje real de los dividendos erogados), es decir, con el otro criterio de aplicación, se observa en los cuadros 9 y 10 que en relación con las mismas variables también existen correlaciones positivas, pero en un nivel considerablemente inferior que las obtenidas con el otro criterio.

\section{Cuadro 9}

Correlación entre el modelo Black y Scholes con el criterio de porcentaje real de los dividendos erogados, $y$ las métricas de creación de valor RION, RSI y UAIIDA

\begin{tabular}{|l|r|r|r|}
\hline & \multicolumn{1}{|c|}{1992} & \multicolumn{1}{c|}{1997} & \multicolumn{1}{c|}{2002} \\
\hline & B\&SDIVPA & B\&SDIVPA & B\&SDIVPA \\
\hline RION\$ & 0.444 & 0.684 & 0.415 \\
\hline RSI\$ & 0.428 & 0.642 & 0.299 \\
\hline UAIIDA\$ & 0.448 & 0.662 & 0.409 \\
\hline
\end{tabular}

Fuente: elaboración propia

\section{Cuadro 10}

Correlación entre el modelo Black y Scholes con el criterio de porcentaje real de los dividendos erogados, y los métodos de valuación de empresas considerados en el estudio

\begin{tabular}{|l|r|r|r|}
\hline & \multicolumn{1}{|c|}{1992} & \multicolumn{1}{c|}{1997} & \multicolumn{1}{c|}{2002} \\
\hline & B\&SDIVPA & B\&SDIVPA & B\&SDIVPA \\
\hline VLIBROS & 0.531 & 0.702 & 0.655 \\
\hline GOODWILL & 0.547 & 0.67 & 0.619 \\
\hline MULT.PU & 0.381 & 0.519 & 0.339 \\
\hline VMERCADO & 0.412 & 0.651 & 0.446 \\
\hline FLUJOSDC & 0.171 & 0.367 & 0.045 \\
\hline
\end{tabular}

Fuente: elaboración propia 
Los métodos de valuación de empresas y su relación con la

capacidad de las organizaciones para generar valor

\section{Propuesta para reportar el valor de la empresa y su capacidad para crear valor}

Dados los resultados observados, la propuesta central de este trabajo es la de contribuir a hacer más clara la información financiera para los tomadores de decisiones. Una forma de hacerla más clara es incluir un valor (además del valor en libros) que informe acerca de lo que la empresa vale en relación con el valor económico que genera o que destruye.

Si se incluyera el valor que la empresa tiene según el método de goodwill tanto operativo como neto se estaría informado si en los últimos cinco años la empresa ha generado el valor económico que se relaciona con la métrica EVA o lo ha destruido. Además, si se incluyera un valor calculado con el modelo Black y Scholes aplicado con el criterio de dividendos equivalente al rendimiento real de la tasa libre de riesgo, se contaría con una información adicional que se relaciona con lo que la empresa puede generar en cuanto al RION, al RSI y al UAIIDA. Como se señaló con anterioridad, este modelo no nos va a dar el valor exacto de la empresa en el futuro, sino que - dadas las características del mismo que con bases probabilísticas sólidas indica el valor que en el futuro podría tener la empresanos señalaría si la empresa fue capaz de generar valor o no.

Si la empresa por medio del modelo Black y Scholes calcula un valor a un año y transcurrido este tiempo se observa que el valor en libros fue superior, entonces se puede concluir que la empresa generó valor, puesto que superó las expectativas probabilísticas que se esperaban. Por el contrario, si se observa que la empresa reporta un valor en libros menor al que las expectativas probabilísticas del modelo nos indicaban, entonces se puede decir que la empresa no alcanzó el valor mínimo que se esperaba de ella en función de las bases probabilísticas del modelo, lo que indicaría que la empresa no generó valor económico que, para este caso, no es el que miden las métricas conocidas, sino que sería un valor en relación con el modelo aplicado, que podría ser llamado valor económico futuro mínimo esperado ${ }^{8}$.

En primer lugar, se observa cómo el valor que arroja este modelo en algunas ocasiones rebasa el valor en libros y en otras tantas se queda por debajo, coincidiendo

\footnotetext{
${ }^{8}$ Ésta es una propuesta de esta investigación doctoral de un modelo de valuación de empresas que pronostica el comportamiento futuro y que puede ser considerado como una métrica de creación del valor económico que mide el propio modelo.
} 
en el 68\% de las ocasiones con el aumento o disminución de las métricas de creación de valor EVA o con las del goodwill porque, como se dijo en el párrafo anterior, es otro valor el que mide. En segundo lugar, se considera que el propio modelo de Black y Scholes puede tomar como referencia el valor en libros porque el propio modelo de Black y Scholes toma las variables que se estudian en este valor y que son los activos como valor del subyacente y los pasivos como valor de ejercicio en la fórmula, así como los dividendos y las variables que hacen del modelo uno de los más acertados para valuar opciones. Estas dos informaciones por separado podrían brindar elementos de análisis importantes para los usuarios de la información financiera.

Con base en estas consideraciones se propone la creación de un nuevo estado financiero anual informativo de la capacidad que la empresa ha tenido para generar valor, el cual se presenta a continuación: 


\section{NOMBRE DE LA EMPRESA \\ Estado de generación de valor al 31 de diciembre de 2004 (miles de pesos)}

\begin{tabular}{lr}
\multicolumn{1}{c}{ Generación de valor } \\
Histórico & \\
Generación de valor neto (1) & $\$ 200,000.00$ \\
Generación de valor operativo (2) & $250,000.00$ \\
& \\
Proyectado & \\
$\begin{array}{l}\text { Valor en libros superior o inferior al mínimo esperado } \\
\text { al cierre del presente año (3) }\end{array}$ & $(100,000.00)$
\end{tabular}

\section{Histórico}

\section{Valor de la empresa}

Valor en libros (4)

$\$ 6,000,000.00$

Valor en función de la generación neta (5)

$6,200,000.00$

Valor en función de la generación operativa (6)

$6,250,000.00$

\section{Proyectado}

Valor mínimo esperado al cierre del presente año (7)

$6,100,000.00$

Valor mínimo esperado al cierre del próximo año (8)

$6,160,000.00$

(1) Valor del goodwill neto

(2) Valor del goodwill operativo

(3) Valor superior o inferior al proyectado por el modelo de Black y Scholes considerando dividendos.

Es la diferencia entre el valor mínimo esperado al cierre del presente año, punto 7, y el valor en libros, punto 4

(4) Valor del capital contable del año

(5) Valor en libros más la generación neta

(6) Valor en libros más la generación operativa

(7) Valor calculado el cierre del año anterior 2003, de lo que se espera a cierre de 2004

(8) Valor calculado al cierre del presente año 2004 de lo que se espera para el cierre de 2005 
En el siguiente estado financiero al 2005 se observa que el valor mínimo esperado para el presente año, punto 7, se calculó y registró en el estado financiero de 2004, punto 8. Como se explicó anteriormente, es un valor proyectado de lo que la empresa podría valer en función del sustento probabilístico que tiene el modelo de Black y Scholes considerando dividendos aplicados en esta investigación. Si el valor en libros es menor, entonces se dice que la empresa tiene un valor inferior al mínimo esperado; pero si es mayor, se dice que tiene un valor superior al mínimo esperado, como se observa en el punto 3. Este valor podría ser llamado como valor económico mínimo esperado como propuesta de esta investigación. 


\section{NOMBRE DE LA EMPRESA}

Estado de generación de valor al 31 de diciembre de 2005 (Miles de pesos)

\section{Generación de valor}

\section{Histórico}

Generación de valor neto (1)

Generación de valor operativo (2)

$$
\$(50,000.00)
$$

Proyectado

\section{Proyectado}

Valor en libros superior o inferior al mínimo esperado al cierre del presente año (3)

\section{Valor de la empresa}

\section{Histórico}

Valor en libros (4)

$\$ 6,200,000.00$

Valor en función de la generación neta (5)

$6,150,000.00$

Valor en función de la generación operativa (6)

$6,300,000.00$

\section{Proyectado}

Valor mínimo esperado al cierre del presente año (7)

$6,160,000.00$

Valor mínimo esperado al cierre del próximo año (8)

$6,450,000.00$

(1) Valor del goodwill neto

(2) Valor del goodwill operativo

(3) Valor superior o inferior al proyectado por el modelo de Black y Scholes considerando dividendos.

Es la diferencia entre el valor mínimo esperado al cierre del presente año, punto 7, y el valor en libros, punto 4

(4) Valor del capital contable del año

(5) Valor en libros más la generación neta

(6) Valor en libros más la generación operativa

(7) Valor calculado el cierre del año anterior 2004, de lo que se espera a cierre de 2005

(8) Valor calculado al cierre del presente año 2005 de lo que se espera para el cierre de 2006 
Con estas señales, los usuarios de la información tendrán más y mejores elementos en relación con el valor de la empresa y con su capacidad para generar valor.

\section{Alternativas para todo tipo de empresas}

A pesar de que la muestra de la investigación sólo es de empresas que cotizan en el mercado de valores, existen alternativas de cálculo para todo tipo de empresas.

Es importante considerar que existen diversas alternativas de aplicación al CAPM para el cálculo del costo promedio ponderado de capital, en especial para empresas que no cotizan en el mercado de valores. A continuación se enlistan estas alternativas:

- Una de las principales alternativas es la que aplica Ma. Luisa Saavedra en $2002^{9}$, que retoma de Haime ${ }^{10}$ y que señala la forma de calcular el costo de capital:

Costo de la deuda de la empresa $=$ Intereses $/$ Pasivo con costo

Costo de deuda de la empresa - Tasa libre de riesgo $=$ Prima de riesgo de la empresa

Costo de capital propio $=$ Costo de la deuda de la empresa + Prima de riesgo de la empresa

- Otra alternativa la presentan Sanjurjo y Reinoso ${ }^{11}$ quienes afirman que para empresas pequeñas el CAPM debería agregar una prima adicional por el reducido tamaño de su mercado. Señalan que la Ibboston Associates, que es una compañía estadounidense establecida en 1977, menciona que aquellas empresas que tengan una capitalización menor a 270 millones de euros, que es donde se ubican la mayoría de las empresas en México, deben aplicar esa prima que es proporcionada por esta empresa, actividad a la que se dedica principalmente.

\footnotetext{
${ }^{9}$ Luis Haime, Reestructuración integral de empresas, México, Isef, 1998, citado por Ma. Luisa Saavedra, La valuación de empresas. Enfoques teóricos y aplicación de los modelos Black y Scholes, Valor Económico Agregado, y flujo de efectivo disponible, p. 95.

${ }^{10}$ Esta alternativa es importante y es aplicable cuando el costo de capital que se obtiene es superior a la tasa libre de riesgo, de lo contrario se tendrá que ajustar a ésta.

${ }^{11}$ Miguel Sanjurjo y Mar Reinoso, Guía de valoración de empresas, p. 312
} 
- Otras alternativas las presenta el autor de esta investigación ${ }^{12}$ en la que las empresas pueden calcular su costo de capital de diversas formas: la tasa libre de riesgo, la inflación y una prima por riesgo calculada sumándole a la tasa libre de riesgo la diferencia entre esa misma tasa y la inflación:

El cálculo del valor económico con estos criterios sería el siguiente para los tres casos:

Rendimiento operativo menos la tasa libre de riesgo

(UO - I / capital inicial) - (Tlr)

Donde: $\mathrm{UO}=\mathrm{Utilidad}$ de operación

$$
\mathrm{I}=\text { ISR y PTU }
$$

Rendimiento operativo menos la inflación

(UO - I / capital inicial) - (inflación)

Rendimiento operativo menos una prima por riesgo sumada a la tasa libre de riesgo menos la inflación

$(\mathrm{UO}-\mathrm{I} /$ capital inicial $)-(\mathrm{T} l \mathrm{r}+(\mathrm{Tl} r-$ inflación $))$

En esta última alternativa, la prima por riesgo representa la diferencia entre lo que ofrece la tasa libre de riesgo y la inflación, que sumada a la propia tasa libre de riesgo representa el doble del rendimiento que ofrece la tasa libre de riesgo sobre la inflación; es decir, por correr riesgo se otorga una ganancia al doble de la que se obtiene por encima de la inflación sin correr riesgo.

- Otra alternativa consiste en utilizar la misma fórmula del CAPM, pero considerando la beta del mercado; es decir, con el valor igual a 1, lo que permite considerar a la empresa que no cotiza con la beta correspondiente al mercado en su conjunto que es 1 .

$$
\begin{aligned}
& \text { CAPM }=\text { Tlr }+\beta(\mathrm{Tm}-\mathrm{Tl}) \\
& \mathrm{CAPM}=\mathrm{Tl} r+(1)(\mathrm{Tm}-\mathrm{Tl})
\end{aligned}
$$

\footnotetext{
${ }^{12}$ Juan Alberto Adam Siade, "Problemática de la aplicación de las métricas de creación de valor en empresas mexicanas", Memoria del VII Foro de Investigación: Congreso Internacional de Contaduría, Administración e Informática, México, Facultad de Contaduría y Administración de la Universidad Nacional Autónoma de México, octubre de 2002, disco compacto, s/p.
} 
- En el caso de la aplicación del modelo de Black y Scholes las empresas tienen a su alcance todos los elementos de la fórmula con excepción de la volatilidad. Se ha observado que la volatilidad no mueve las cifras en forma considerable. Una alternativa que tienen las empresas es la de tomar la volatilidad promedio del mercado o, incluso, la volatilidad del sector al que las mismas pertenecen.

\title{
5. Análisis de la normatividad financiera mexicana e internacional en función de la necesidad de reportar, en la información financiera, el valor de las organizaciones y la capacidad que tienen para crearlo
}

La información financiera contenida en los estados financieros podría modificarse de diversas formas para ser más clara y mejor. Este trabajo se limita exclusivamente a hacer un análisis de cómo reportar el valor de la empresa y su capacidad para generarlo puede contribuir a lograr en forma más adecuada el cumplimiento de la normatividad contable. Asimismo, se refiere sólo a las normas internacionales y a la normatividad mexicana en materia contable.

\section{A. Negocio en Marcha}

La Norma Internacional de Contabilidad 1 (NIC en adelante), en lo que respecta al apartado referente a Negocio en Marcha, nos señala lo siguiente:

\begin{abstract}
Al preparar los estados financieros, la gerencia debe realizar una evaluación sobre la posibilidad de que la empresa continúe en funcionamiento. Los estados financieros deben prepararse a partir de la suposición de Negocio en Marcha, a menos que la gerencia, o bien pretenda liquidar la empresa o cesar en su actividad, o bien no exista una alternativa realista de continuación salvo que proceda de una de estas formas. Cuando la gerencia, al realizar esta evaluación, sea consciente de la existencia de incertidumbres importantes, relativas a sucesos o condiciones que pueden aportar dudas significativas sobre la posibilidad de que la empresa siga funcionando normalmente, debe revelarlas en los estados financieros. En el caso de que los estados financieros no se preparen sobre la base de Negocio en Marcha, tal hecho debe ser objeto de revelación explicita, junto con las hipótesis alternativas sobre las que han sido elaborados y las razones por las que la entidad no puede ser considerada como un Negocio en Marcha. ${ }^{13}$
\end{abstract}

\footnotetext{
${ }^{13}$ Con base en International Accounting Standards Board e Instituto Mexicano de Contadores Públicos, Normas internacionales de información financiera, septiembre de 2003, pp. 1-15.
} 
Por su parte el boletín A1 de Principios de Contabilidad Generalmente Aceptados (PCGA en adelante), editado por el Instituto Mexicano de Contadores Públicos (IMCP en adelante), nos indica lo siguiente:

La entidad se presume en existencia permanente, salvo especificación en contrario; por lo que las cifras de sus estados financieros representarán valores históricos, o modificaciones de ellos, sistemáticamente obtenidos. Cuando las cifras representan valores estimados de liquidación, esto deberá especificarse claramente y solamente serán aceptables para información general cuando la entidad esté en liquidación. ${ }^{14}$

\section{- Análisis y comentarios referentes al Negocio en Marcha $^{15}$}

Se considera que esta norma es importante porque la esencia de las entidades es permanecer, puesto que no fueron creadas para desaparecer. En este sentido, garantizar la permanencia de un negocio es mantener los bienes y servicios que comercializa, produce y ofrece, así como los empleos que se generan y que se suman a todos los beneficios económicos que trae consigo la existencia permanente de una organización.

Como los estados financieros se presentan actualmente con cifras históricas, le permiten a la gerencia determinar si el negocio podrá seguir en marcha o no. Simplemente si el historial de la entidad ha sido bueno y no se vislumbra que habrá un acontecimiento que pueda interrumpir su continuidad, se puede concluir que, cuando menos, por uno o algunos periodos más el negocio continuará. Esto es suficiente para que la información financiera se presente bajo la base de Negocio en Marcha.

Sin embargo, se considera que la información contenida en los estados financieros podría ser más financiera; es decir, podría ser más completa y mejor en relación con lo que las normas deberían entender por Negocio en Marcha y que, por lo que se lee, no lo expresan claramente porque hablan sólo de las cifras contenidas en los estados financieros que son históricas y porque no establecen la forma en que la gerencia podrá determinar las condiciones financieras en las que el negocio continuará.

\footnotetext{
${ }^{14}$ Instituto Mexicano de Contadores Públicos, Principios de Contabilidad Generalmente Aceptados, 2003, s/p.

${ }^{15}$ En el desarrollo de este trabajo se utilizarán los términos norma y principio indistintamente dado que internacionalmente se llaman normas, pero en México se conocen como principios, sin ser motivo de este estudio la discusión entre la pertinencia del uso de uno o de otro.
} 
Se considera que si hablamos de Negocio en Marcha no es suficiente conocer las cifras históricas, puesto que ellas sólo nos indican lo que en el pasado sucedió. Hablar de Negocio en Marcha es el antes y el después; es hablar de lo que la empresa ha sido, pero también lo que la empresa será. Negocio en Marcha no sólo puede ser el pasado en números, sino que también el futuro. Además, el principio de Negocio en Marcha se ha orientado exclusivamente a decir que la gerencia determinará si la entidad continuará o no, pero financieramente se ha limitado porque lo importante no sólo es saber si continuará, sino en qué condiciones lo hará.

Incluir el valor de la empresa en los estados financieros y la capacidad que ésta tiene para generar valor con los métodos que se aplican al pasado y que se adelantan al futuro financiero de la entidad contribuirán a que el principio de Negocio en Marcha se cumpla de forma más adecuada.

Se considera que con esta información, derivada de la propuesta presentada en el capítulo anterior, los estados financieros se prepararán con bases más sólidas sobre la suposición de Negocio en Marcha; la gerencia tendrá elementos más sólidos para determinar no sólo si la empresa permanecerá, sino además en qué condiciones lo hará, lo que le permitirá tomar decisiones correctivas en caso de que se presente un panorama desfavorable.

Este análisis continúa con el concepto de desempeño que forma parte de los elementos de los estados financieros dentro del marco conceptual de las Normas Internacionales de Información Financiera.

\section{B. Elementos de los estados financieros}

Dentro del marco conceptual para la preparación y presentación de los estados financieros, documento aprobado por el Consejo del Comité de las Normas Internacionales de Contabilidad (IASC por sus siglas en inglés) en abril de 1989 y vigente hasta la fecha, se establece como elemento de los estados financieros el desempeño.

Desempeño en este marco conceptual se refiere a lo siguiente: 
La cifra del resultado es a menudo usada como una medida del desempeño en las actividades de la empresa, o bien es la base de otras evaluaciones, tales como el rendimiento de las inversiones o las ganancias por acción. Los elementos relacionados directamente con la medida del resultado son los ingresos y los gastos. El reconocimiento y medida de los ingresos y gastos, y por lo tanto del resultado, dependen en parte de los conceptos de capital y mantenimiento del capital usados por la empresa al elaborar los estados financieros. ${ }^{16}$

\section{- Análisis y comentarios en relación con el desempeño}

La normatividad internacional, en este sentido, no considera cuestiones más importantes que los resultados obtenidos por la diferencia entre los ingresos y los gastos para medir el desempeño de las empresas. Para los usuarios de la información sería más importante que, además de indicarles cuál es el resultado contable, se les dijera en qué medida la empresa está generando o no valor en función de diferentes factores. Que además de conocer el valor del capital usado por la empresa, que representa el valor en libros, se les dijera cuánto vale la misma en función de la capacidad que tiene y que tendrá para generar valor.

Con esto, la propuesta de esta investigación contribuye a que la información financiera permita tener una medida de desempeño basada no sólo en el resultado contable que como tal, en estos momentos y dadas las necesidades que requieren los inversionistas y los usuarios de la información financiera en general, no aporta mucho para la toma de decisiones.

Por su parte, la normatividad mexicana en cuanto a los PCGA no establece este concepto dentro de los elementos básicos de los estados financieros ${ }^{17}$, que sería valioso como elemento por considerar dentro de los beneficios que puede brindar la información financiera.

Este análisis continúa con el apartado de características cualitativas de los estados financieros en relación con la relevancia de la información.

\footnotetext{
16 International Accounting Standards Board, op. cit., p. M-28.

17 Véase el boletín A-11 de los PCGA, "Definición de los conceptos básicos integrantes de los estados financieros".
} 


\title{
C. Características cualitativas de los estados financieros
}

Dentro del mismo marco conceptual para la preparación y presentación de los estados financieros se establece como característica cualitativa de los estados financieros el concepto de relevancia que dice:

\begin{abstract}
Para ser útil, la información debe ser relevante de cara a las necesidades de toma de decisiones por parte de los usuarios. La información posee la cualidad de la relevancia cuando ejerce influencia sobre las decisiones económicas de los que la utilizan, ayudándoles a evaluar sucesos pasados, presentes of futuros, o bien a confirmar o corregir evaluaciones realizadas anteriormente. ${ }^{18}$
\end{abstract}

En relación con la relevancia, la normatividad mexicana en el boletín A-1, "Esquema de la teoría básica de la contabilidad financiera”, señala lo siguiente:

La utilidad de la información está en función de su contenido informativo y de su oportunidad. El contenido informativo está basado en: [...] la relevancia de la información que es la cualidad de seleccionar los elementos de la misma que mejor permitan al usuario captar el mensaje y operar sobre ella para lograr sus fines particulares; la veracidad [... $]^{19}$

\section{- Análisis y comentarios referentes a la relevancia de la información}

Como podemos observar, tanto la norma internacional como la mexicana fundamentan la relevancia en función de la utilidad que la información le proporciona al usuario; también se observa que la internacional es más completa, clara y explícita, lo que permite tener más elementos de juicio para poder hacer un análisis.

Como se ha mencionado, la información contenida en los estados financieros de la entidad sólo se refiere a los eventos que ya han sucedido, es decir, que ya pasaron. En este sentido poco nos dice esta información que sin duda es relevante; sin embargo, sólo nos sirve para evaluar sucesos pasados y presentes en forma acertada, pero en forma limitada los sucesos futuros.

La aportación de este trabajo contribuye a que se incluya como información relevante el valor de la empresa en función de lo que ha realizado y de lo que realizará en términos razonables, así como la capacidad que ha tenido, que tiene y que

\footnotetext{
${ }_{18}$ International Accounting Standards Board, op. cit., p. M-15.

${ }^{19}$ Instituto Mexicano de Contadores Públicos, Principios de contabilidad generalmente aceptados, [s.p.]
} 
tendrá para generar valor; con ello se logrará que la información posea una mayor calidad de relevancia que ayude a evaluar en forma más amplia los sucesos futuros, además de los pasados y los presentes. Con esto también se logrará que el contenido de la información sea más importante al ser más selecta, lo que le permitirá a los usuarios de la misma tener más elementos para lograr sus fines particulares.

Como se puede observar, la propuesta de este trabajo contribuye a que la información financiera sea más selecta y, por lo tanto, más relevante para sus usuarios.

\section{Importancia relativa}

Según la NIC-1, la importancia relativa o material la presenta como parte esencial del concepto de relevancia al señalar lo siguiente:

La relevancia de la información está afectada por su naturaleza e importancia relativa.

La información tiene importancia relativa, o es material, cuando su omisión o presentación errónea pueden influir en las decisiones económicas de los usuarios, tomadas a partir de los estados financieros [...] De esta manera, el papel de la importancia relativa es suministrar un umbral o punto de corte, más que ser una característica cualitativa primordial que la información ha de tener para ser útil. ${ }^{20}$

Por su parte, la normatividad mexicana señala tanto en el boletín A-1 como en el A-6, "Importancia relativa", lo siguiente:

La información que aparece en los estados financieros debe mostrar los aspectos importantes de la entidad susceptibles de ser cuantificados en términos monetarios. Tanto para efectos de los datos que entran al sistema de información contable como para la información resultante de su operación, se debe equilibrar el detalle y multiplicidad de los datos con los requisitos de utilidad y finalidad de la información. ${ }^{21}$

\section{- Análisis y comentarios sobre la importancia relativa}

Los aspectos importantes de la entidad susceptibles de ser cuantificados en términos monetarios también los representa la capacidad que la empresa tiene para

\footnotetext{
${ }^{20}$ International Accounting Standards Board, op. cit., p. M-16.

21 Instituto Mexicano de Contadores Públicos, op.cit., [s.p.]
} 
generar valor o para destruirlo. En este sentido, la propuesta de esta investigación contribuye a que este principio se cumpla en forma más adecuada porque la capacidad que la empresa tiene para generar valor es un dato que constantemente se puede conocer. Soslayar esta información o simplemente no considerarla representaría omitir datos importantes para la toma de decisiones de los usuarios de la información financiera, que están en forma implícita en la empresa, pero que hay que mostrar en forma explícita.

Es evidente que teniendo estos avances teóricos en las finanzas tenemos más elementos por considerar para la toma de decisiones. El omitir estos datos va en contra de este principio que podría cumplirse en forma más adecuada mejorando la calidad de la información contenida en los estados financieros, a través de la capacidad que las empresas tienen para crear o no valor.

\section{Conclusiones}

A raíz de los resultados observados, el método goodwill — que ha sido considerado sólo como un método de valuación de empresas-, además de permitirnos calcular el valor de las empresas, también puede ser aplicado como una métrica de la generación de valor de las mismas que se relaciona con el EVA, puesto que si presenta goodwill positivo la empresa genera valor en la proporción que corresponda al resultado; pero si presenta goodwill negativo, la empresa está destruyendo valor en la proporción que le corresponda al resultado.

Dadas las características del método mencionado, sus resultados se pueden manejar en dos etapas:

En la primera se va a determinar si la empresa presentó goodwill positivo o negativo para, en función de ello, saber si generó o destruyó valor económico. En esta primera etapa se determina el porcentaje de creación o de destrucción de valor y, por lo tanto, se considera (como una de las propuestas de esta investigación) una métrica de la generación de valor de la empresa.

En la segunda etapa vamos a determinar el valor de la empresa sumándole ese valor del goodwill a los activos netos como lo dicta la teoría para, entonces, considerarlo método de valuación de empresas. 
Como métrica de creación de valor, se relaciona con el EVA más que con la GEO, que se calcula con los mismos criterios que el propio EVA, y más que con el RION, el RSI, la UAIIDA y el rendimiento bursátil.

Esto significa que si una empresa está siendo valuada para efectos de una venta, compra, fusión, escisión o alianza, el valuador va a tener elementos de creación de valor importantes cuando aplique el método goodwill, que es similar, que se relacionan y que presentan la misma tendencia que los resultados que se obtienen con el EVA.

El EVA es una marca registrada por Stern Stewart \& Co. Las empresas que lo han adoptado como métrica de creación de valor han tenido que pagar derechos por el uso de la marca; por otro lado, el goodwill es de dominio público, por lo que las empresas lo pueden aplicar como métrica de creación de valor libremente, con una ventaja sobre el EVA, ya que este método sólo mide la capacidad que la empresa tuvo para generar valor en el último año, mientras que el goodwill lo hace con relación a la capacidad que la empresa tuvo para generarlo en los últimos cinco.

Por otra parte, si en los estados financieros se incluyera el valor que la empresa tiene según el método de goodwill, tanto el relacionado con el rendimiento operativo como con el neto, se estaría informado si en los últimos cinco años la empresa ha generado el valor económico, que se relaciona con la métrica EVA, o lo ha destruido.

En una valuación es tan importante considerar la historia de la empresa como su posible futuro; en este sentido, si además se incluyera en un proceso de negociación un valor calculado con el modelo Black y Scholes aplicado con el criterio de dividendos equivalente al rendimiento real de la tasa libre de riesgo, los empresarios y los ejecutivos contarían con una información adicional que se relaciona con el valor que la empresa podría generar en el futuro que se relaciona con el RION, al RSI y al UAIIDA.

Este método Black y Scholes no nos va a brindar el valor exacto que la empresa tendrá en el futuro, sino que, dadas las características del mismo que con bases probabilísticas sólidas indica el valor que en el futuro podría tener la empresa, nos señalaría si la empresa fue capaz de generar valor o no en relación con el valor que de ella misma se esperaba. 
Si el modelo de Black y Scholes, calculado con el criterio mencionado, determina el valor que una empresa tendrá en un año y se observa que después de ese año el valor en libros rebasó la expectativa, es decir que fue superior, se puede concluir que la empresa generó valor. Por el contrario, si el valor en libros no alcanzó las expectativas, es decir, que se quedó por debajo del valor pronosticado, se podría concluir que la empresa no generó valor. En este caso no sería un valor relacionado con las métricas conocidas, sino que sería una forma más como la empresa puede medir la creación de valor. En este caso podría llamarse valor económico futuro mínimo esperado.

El valor que calcula este modelo en algunas ocasiones rebasa el valor en libros y en otras se queda por debajo, coincidiendo en casi el 70\% de las ocasiones con el aumento o disminución de las métricas de creación de valor EVA o con las del goodwill (porque como se dijo es otro valor el que mide). Se puede tomar como referencia el valor en libros porque el propio modelo de Black y Scholes toma las variables que se estudian en dicho valor: los activos como valor del subyacente y los pasivos como valor de ejercicio en la fórmula.

Se observaron resultados importantes y dignos de ser analizados en investigaciones posteriores referentes al rendimiento bursátil y su relación con algunos de los métodos y métricas analizados en esta investigación, que podrían dar indicios de una relación entre el análisis fundamental con el rendimiento bursátil futuro, lo que podría tener alguna relación con el valor intrínseco de la acción.

La propuesta de esta investigación de crear un estado financiero nuevo llamado Estado de Generación de Valor que integra el valor de la empresa en función de la generación histórica de valor y proyectada, puede contribuir a cumplir en forma más adecuada la normatividad contable mexicana e internacional en lo que respecta al Negocio en Marcha, la Revelación Suficiente, la Importancia Relativa y en términos generales al desempeño de la empresa. Asimismo, puede brindar elementos de análisis importantes para los usuarios de dicha información. De igual forma, esta propuesta le brinda a los inversionistas y a todos los usuarios de la información elementos que los estados financieros tradicionales no contienen como es el caso de la capacidad que la empresa ha tenido y tendrá para generar valor.

Esta propuesta al incluir el valor que de la empresa se espera tener en el futuro cambia el paradigma en relación con los números que encontramos en los estados financieros que, como se presentan, sólo representan los acontecimientos pasa- 
dos. También existen diversas alternativas para que las diferentes empresas puedan aplicar estas métricas y este tipo de técnicas.

Los trabajos futuros que se desprendan de esta investigación en relación con la línea de investigación de valuación de empresas y creación de valor estarán enfocadas a lo siguiente:

- Explorar la normatividad nacional e internacional en lo referente a la valuación de los activos intangibles.

- Estudiar la importancia del valor intrínseco de una acción en relación con la influencia del análisis fundamental en el comportamiento bursátil, en el mediano y largo plazos.

\section{Bibliografía}

Adam Siade, Juan Alberto, “Análisis de la valuación del rendimiento financiero de las empresas en función del CAPM y del WACC en mercados emergentes: exploración sobre las ventajas y desventajas de emplear una segunda beta", en Memoria, $V$ Foro Nacional de Investigación en las Disciplinas Financiero Administrativas, México, Facultad de Contaduría y Administración, UNAM, octubre de 2000, disco compacto, s/p.

-, "La problemática de la aplicación de los modelos CAPM y WACC en mercados emergentes", en Contaduría y Administración, $\mathrm{N}^{\circ} .198$, julioseptiembre de 2000, México, FCA-UNAM, pp. 5-10.

, "La reexpresión de estados financieros en la valuación de acciones", en Contaduría y Administración, №.193, abril-junio de 1999, México, FCA-UNAM, pp. 25-35.

, "Problemática de la aplicación de las métricas de creación de valor en empresas mexicanas", en Memoria, VII Foro de Investigación: Congreso Internacional de Contaduría, Administración e Informática, México, Facultad de Contaduría y Administración, UNAM, octubre de 2002, disco compacto, [s.p.] 
, "Valuación de empresas con los métodos de valor en libros, de mercado, múltiplo precio utilidad, goodwill y Black y Scholes", en Contaduría y Administración, $\mathrm{N}^{\circ} .182$, julio-septiembre de 1996, México, FCA-UNAM, pp. 33-42.

et al., "Panel de finanzas", en Memoria, II Foro Nacional de Investigación, México, Facultad de Contaduría y Administración, UNAM, octubre de 1997, pp. 110-143.

, Héctor M. de Uriarte, Francisco Ibáñez Cortina y Manuel Mena Zárate (coordinadores), Valuación de empresas y creación de valor, México, UNAM-PricewaterhouseCoopers-IMEF, 2002, 270 pp.

Alvarado Martínez de Escobar, Lourdes, La contaduría pública, México, UNAM, 1983, 144 pp.

Álvarez Plaza, José Jaime, "Valoración de empresas: intangibles y tecnología de información”, en Memoria, VIII Foro de Investigación, Congreso Internacional de Contaduría, Administración e Informática, México, Facultad de Contaduría y Administración, UNAM, octubre de 2003, disco compacto, s/p.

American Society of Appraisers, Business valuation: introduction to business valuation, Parte 1, v. 1.0s (3/98), EEUU, American Society Of Appraisers, 1998.

Bae, Kee-Hong, Jun-Koo Kang y Jin-Mo Kim, “Tunneling or value added? evidence from mergers by korean business groups", The journal of finance, vol. 57, diciembre de 2002, EEUU, The American Finance Association, pp. 26952740 .

Benninga, Simon Z. y Oded H. Sarig, Corporate finance a valuation approach, EEUU, McGraw-Hill, 1997, 445 pp.

Besley, Scott y Eugene F. Brigham, Fundamentos de administración financie$r a$, decimosegunda edición, México, McGraw-Hill, 2001, 919 pp.

Black, Andrew, Phillip Wright y John Davis, In search of shareholder value, segunda edición, Prentice, 2001, 306 pp. 
Black, Fisher y Miron Scholes, The valuation of option contracts and a test of market efficiency, Journal of Finance 27, EEUU, 1972, pp. 399-417. Political Economy, volumen 81, número 3, mayo/junio, Universidad de Chicago, Chicago, 1973.

Bolsa Mexicana de Valores, Anuarios financieros, 1987-2002.

, Anuarios bursátiles, 1987-2002.

-, "Emisoras", México, www.bmv.com.mx, 10 de junio de 2004.

Brealey, Richard A. y Stewart C. Myers, Pincipios de finanzas corporativas, cuarta edición, Madrid, McGraw-Hill, 1996, 1203 pp.

Cabello Rosales, Alejandra, El modelo Black y Scholes y sus modificaciones. México, notas en preparación, 2004.

Damodaran, Aswath, Damodaran on valuation: security analysis for investment and corporate finance, EEUU, John Wiley \& Sons, 1994, 426 pp.

, Investment valuation, EEUU, John Wiley \& Sons, 1996.

Day, Alastair L., Mastering financial modeling, 1a. ed., Londres, Financial Times Prentice Hall, Pearson Education, 2001, 370 pp.

Eccles, Robert G., Robert H. Herz, E. Mary Keegan y David M. H. Phillips, The value reporting revolution, PricewaterhouseCoopers, Nueva York, 2001, 349. pp.

Díaz Mata, Alfredo, Invierta en la bolsa, segunda edición, México, Grupo Editorial Ibérica, 1994, 307 pp.

Diez de Castro, Luis y Juan Mascareñas Pérez-Íñigo, Ingeniería financiera, segunda edición, Madrid, McGraw-Hill, 1994, 467 pp.

Easley, David y Maureen O'Hara, "Information and the cost of capital", The journal of finance, vol. 59, agosto de 2004, EEUU, The American Finance Association, pp. 1553-1583. 
Evans, Frank C. y David M. Bishop, Valuation for $M \& A$ : building value in private companies, EEUU, John Wiley \& Sons, 2001, 298 pp.

Ferrater Mora, José, Diccionario de filosofia, Barcelona, Ariel, 2001, 4 tomos, $3830 \mathrm{pp}$.

Fishman, Jay E. et al., Guide to business valuations: volumen 1, séptima edición, Forth Worth, Texas, Practitioners Publishing Company, 1997.

García Meca, Emma y Salvador Marín Hernández, "El capital intelectual y la valoración de las grandes empresas españolas", en Memoria, VIII Foro de Investigación: Congreso Internacional de Contaduría, Administración e Informática, Facultad de Contaduría y Administración, UNAM, México, octubre de 2003, disco compacto, s/p.

Helfert, Erich, Valuación, México, ECASA, 1981, 167 pp.

Hodgson, Douglas J. y Keith Vorkink, "Asset pricing Theory and the valuation of canadian paintings", Canadian Journal of Economics, vol. 37, agosto de 2004, University of Rochester, Brigham Young University, J. Willard and Alice S. Marriott School, pp. 629-655.

Ibbotson Associates, Stocks, bonds, bills and inflation: sbbi valuation edition 1999 yearbook, Chicago, Illinois, Ibbotson Associates, 1999, 272 pp.

Instituto Mexicano de Contadores Públicos y ANFECA, Principios de contabilidad generalmente aceptados, decimoctava edición, México, IMCP, 2003, $678 \mathrm{pp}$.

International Accounting Standards Board e Instituto Mexicano de Contadores Públicos, Normas internacionales de información financiera, México, IASB, IMCP, 2003, [s.p.]

Jegadeesh, Narasimhan et al., "Analyzing the analysts: when do recommendations add value?", The journal of finance, vol. 59, junio de 2004, EEUU, The American Finance Association, pp. 1083-1124. 
Kohler, Heinz, Estadística para negocios y economía, México, CECSA, 1999, $1053 \mathrm{pp}$.

La Porta, Rafael et al., "Investor protection and corporate valuation", The journal offinance, vol. 57, junio de 2002, EEUU, The American Finance Association, pp. 1147-1170.

Lemmon, Michael I. y Karl V. LINS, "Ownership structure, corporate governance, and firm value: evidence from the east asian financial crisis", The journal of finance, vol. 58, agosto de 2003, EEUU, The American Finance Association, pp. 1445-1468.

Lev, Baruch, "Sacar más ventaja de los intangibles", Harvard Business Review, vol. 82, N. 6, junio de 2004, pp. 99-106.

Mc Taggart, James M., Peter W. Kontes, y Michael C. Mankins, The value imperative: managing for superior shareholders, EEUU, The free press, 1994, 367 pp.

Meigs, Robert F. et al., Contabilidad. La base para decisiones gerenciales, décima edición, Santa Fe de Bogotá, McGraw-Hill, 1999, 881+XIII pp.

Moreno Fernández, Joaquín, Las finanzas en la empresa, quinta edición, México, Instituto Mexicano de Contadores Públicos, 1994, 580 pp.

Ochoa Torres, Miguel, Guillermo Quiroz Abed y Raúl Velarde Dabrowski, Rion, geo y valore, tres instrumentos para generar valor, México, IPADE, 1997, $110 \mathrm{pp}$.

Pratt, Shannon P.,Cost of capital: estimation and applications, EEUU, John Wiley \& Sons, Inc., 1998, 226 pp.

, Robert F. Reilly y Robert P. Schweihs, Valuing a business: the analysis and appraisal of closely held companies, tercera edición, EEUU, Richard D. Irwin, 1996, 850 pp. 
Rappaport, Alfred, Creating shareholder value, the new standard for business performance, Free press, 1986.

Read, Nick, y Jonathan Batson, Spreadsheet modelling best practice, Londres, Business Dynamics and PricewaterhouseCoopers, 1999, 204 pp.

Real Academia Española, Diccionario de la lengua española, vigesimosegunda edición, Madrid, Espasa Calpe, 2001.

Rodríguez de Castro, J., Introducción al análisis de productos financieros derivados, México, Limusa, 1995, 258 pp.

Ross, Stephen A., Randolph W. Westerfield y Jeffrey F. Jaffe, Finanzas corporativas, quinta edición, México, Irvin McGraw-Hill, 2000, 1053 pp.

Saavedra García, María Luisa, La valuación de empresas. Enfoques teóricos y aplicación de los modelos Black y Scholes, valor económico agregado, y flujo de efectivo disponible en México: 1991-2000, Tesis doctoral inédita, Universidad Nacional Autónoma de México, Facultad de Contaduría y Administración, División de Estudios de Posgrado, México, 2002, 175 pp.

Sanjurjo Miguel y Mar Reinoso, Guía de valoración de empresas, segunda edición, Madrid, PricewaterhouseCoopers-Prentice Hall, 2003, 682 pp.

Saunders, Anthony, Financial institutions management, tercera edición, EEUU, Irwin McGrawHill, 1999.

Sheth, Jagdish, y Rajendra Sisodia, The rule of three, surviving and thriving in competitive markets, Free Press, 2002.

Siu Villanueva, Carlos, Valuación de empresas, México, IMCP, 2001, 230 pp.

Smith, Gordon V., Trademark valuation, EEUU, John Wiley \& Sons, 1997, $293 \mathrm{pp}$.

Solnik, Bruno, Inversiones internacionales, EEUU, Adisson-Wesley Iberoamericana, 1991, $457 \mathrm{pp}$. 
Stern, Joel M. y John S. Shiely, The EVA challenge, implementing value-added change in an organization, Wiley, 2001.

Stewart III, G. Bennett, The quest for value, EEUU, Harper Business, 1991, $781 \mathrm{pp}$.

The Appraisal Foundation, Uniform Standards of Professional Appraisal Practice: 1999 Edition, EEUU, The Appraisal Foundation, 1999, 164 pp.

Ulrich, Dave y Norm Smallwood, "Capitalizar las capacidades", Harvard Business Review, vol. 82, №. 6, junio de 2004, pp. 108-115.

Van Horne, James C., Administración financiera, novena edición, México, Prentice Hal, 1993.

Wang, Kevin Q., "Asset pricing with conditioning information: a new test”, The journal of finance, vol. 58, febrero de 2003, EEUU, The American Finance Association, pp. 161-196. 